\section{Providing support for leading} South African dental institutes

GC UK, in association with Henry Schein Dental Warehouse, is delighted to have been able to lend its support to four leading South African dental institutes, with a donation to each of 50 packs of GC Fuji IX GP, one of the world's leading glass ionomer restoratives.

This much appreciated and welcome material contribution will be used to support dental teaching and clinical skills programmes at the University of Pretoria, University of Witwatersrand, Sefako Makgatho Health Sciences University and the University of Western Cape.

This follows on from another recent GC and Henry Schein Dental donation of 30 packs of GC Fuji IX to the pioneering dental charity Dentaid. This donation is currently being used by Dentaid's volunteer teams working in Uganda, Cambodia, Malawi and Kenya, as they continue to run outreach dental clinics in some of the poorest and most remote regions of the world, taking basic dental services to communities where there is no other access to safe dental care.

GC Fuji IX GP is ideal for permanent or temporary Class I and II restorations in children's teeth, non-load-bearing Class I and II restorations in adult teeth, and Class V and root surface restorations. It is also ideally suited for core build-up and for use with a composite or an inlay in the immediate or delayed sandwich technique. This flexibility and wide range of applications make the material perfect for a teaching environment, enabling tomorrow's young clinicians to become familiar with a host of different techniques.

John Maloney, GC Country Manager for the UK, Ireland and South Africa made the presentation of Fuji IX GP in person together with senior members of the Henry Schein Dental Warehouse team to leading representatives from all four universities.

To find out more about Fuji IX contact GC on +441908218999 / +27824573200, email info.uk@gc.dental, visit www.gceurope.com or contact Henry Schein Dental Warehouse TOLLFREE 0800100796.

\title{
New microsite provides safe and effective treatment options
}

GC Europe has launched Safe4Dentistry.com, a resource centre for dental professionals on mitigating risks of aerosols during treatment.

Recognising how dramatically the COVID-19 pandemic has impacted the practice of dentistry, GC has developed a microsite dedicated to providing the latest updates on evolving guidelines for dental professionals. Safe4Dentistry.com is a continually updated resource centre that provides safe and effective minimally invasive dental treatment options and data, including information on how to reduce aerosol production to protect staff and patients during appointments.

The Safe4Dentistry.com site is populated with podcasts, clinical case examples, continuing education (CE) courses, and access to live webinars, recent articles and other resources. Topics include placing minimally invasive low-aerosol fillings, how to approach emergency treatments, ways to keep the dental practice safe and secure, and much more.

Minimally invasive restoration techniques often eliminate the need for high-speed drilling, which creates aerosols that can impact both patients and practitioners. Using glass ionomer and glass hybrid restorative technology systems provides interim and long-term restorative solutions. Featured cases demonstrate how blending glass hybrids and traditional materials and methods can reduce aerosols, such as utilising materials like EQUIA Forte HT glass hybrid restoratives that require no highspeed drilling or extensive tooth preparation.

Implementing the correct protocols is paramount to patient and staff safety but keeping track of the rapidly changing recommendations and guidelines can be daunting. The Safe and Secure Practice section of the microsite contains the latest updates on evolving guidelines by organisations such as WHO, FDI, and many other European associations, step-bystep procedures, and relevant articles and resources for the dental practitioner.

The Additional Resources section contains systematic reviews, additional tutorials, webinars and podcasts. Presenters include Prof Dr Ivana Miletić, Prof Dr Sevil Gürgan, Prof Dr Sebnem Türkün, Dr José Ignacio Zalba Elizari, Dr John Nicholson and many others. Dental professionals can also request online training and in office visits with their local representatives.

GC Europe is committed to the safety and wellbeing of both oral health providers and patients and the quality of its products. To gain user opinions and suggestions, Safe4Dentistry. com also includes tools to collect the opinions of our customers on certain topics.

For more information, visit http://Safe4Dentistry.com/.

\section{Special protection from acid erosion}

Arm \& Hammer has been a trusted brand for more than 170 years and is committed to helping your patients achieve better oral health with specially formulated low abrasive toothpastes.

Arm \& Hammer Enamel Repair toothpaste repairs and strengthens the enamel surface in five days for healthier teeth. This clinically proven and advanced baking soda formula provides outstanding cleaning and removes more plaque around the gumline than regular toothpastes.

The patented Arm \& Hammer Liquid Calcium technology fills tooth crevices to restore tooth enamel and protect tooth surfaces from acid erosion. For a toothpaste that cleans, restores and protects, contact Arm \& Hammer for more details.

For more information about the carefully formulated Arm \& Hammer toothpaste range, visit http://www. armandhammer.co.uk/ or email: ukenquiries@churchdwight.com.

Arm \& Hammer oral healthcare products are available at Boots, Superdrug, Sainsbury's, Tesco, Asda and Morrisons throughout the UK. 